\title{
LOCALIZATION FOR AN ANDERSON-BERNOULLI MODEL WITH GENERIC INTERACTION POTENTIAL
}

\author{
HAKIM BOUMAZA
}

\begin{abstract}
We present a result of localization for a matrix-valued AndersonBernoulli operator, acting on $L^{2}(\mathbb{R}) \otimes \mathbb{R}^{N}$, for an arbitrary $N \geq 1$, whose interaction potential is generic in the real symmetric matrices. For such a generic real symmetric matrix, we construct an explicit interval of energies on which we prove localization, in both spectral and dynamical senses, away from a finite set of critical energies. This construction is based upon the formalism of the Fürstenberg group to which we apply a general criterion of density in semisimple Lie groups. The algebraic nature of the objects we are considering allows us to prove a generic result on the interaction potential and the finiteness of the set of critical energies.
\end{abstract}

\section{INTRODUCTION}

In this article, we will discuss a generic result on localization properties for the following random family of quasi one-dimensional Anderson-Bernoulli operators :

$$
H_{\ell}(\omega)=-\frac{\mathrm{d}^{2}}{\mathrm{~d} x^{2}} \otimes I_{\mathrm{N}}+V+\sum_{n \in \mathbb{Z}}\left(\begin{array}{ccc}
c_{1} \omega_{1}^{(n)} \mathbf{1}_{[0, \ell]}(x-\ell n) & 0 \\
& \ddots & \\
0 & & c_{N} \omega_{N}^{(n)} \mathbf{1}_{[0, \ell]}(x-\ell n)
\end{array}\right),
$$

acting on $L^{2}(\mathbb{R}) \otimes \mathbb{R}^{N}$, where $N \geq 1$ is an integer, $I_{\mathrm{N}}$ is the identity matrix of order $N$ and $\ell>0$ is a real number. The matrix $V$ is a real $\mathrm{N} \times \mathrm{N}$ symmetric matrix, the space of these matrices being denoted by $\mathrm{S}_{\mathrm{N}}(\mathbb{R})$. The constants $c_{1}, \ldots, c_{N}$ are non-zero real numbers.

For every $i \in\{1, \ldots, N\}$, the $\left(\omega_{i}^{(n)}\right)_{n \in \mathbb{Z}}$ are sequences of independent and identically distributed i.i.d. random variables on a complete probability space $(\widetilde{\Omega}, \widetilde{\mathcal{A}}, \widetilde{\mathrm{P}})$, of common law $\nu$ such that $\{0,1\} \subset \operatorname{supp} \nu$ and supp $\nu$ is bounded. In particular, the $\omega_{i}^{(n)}$ 's can be Bernoulli random variables. The family $\left\{H_{\ell}(\omega)\right\}_{\omega \in \Omega}$ is a family of random operators indexed by the product space

$$
(\Omega, \mathcal{A}, \mathrm{P})=\left(\otimes_{n \in \mathbb{Z}} \widetilde{\Omega}^{\otimes N}, \otimes_{n \in \mathbb{Z}} \widetilde{\mathcal{A}}^{\otimes N}, \otimes_{n \in \mathbb{Z}} \widetilde{\mathrm{P}}^{\otimes N}\right) .
$$

We also set, for every $n \in \mathbb{Z}, \omega^{(n)}=\left(\omega_{1}^{(n)}, \ldots, \omega_{N}^{(n)}\right)$, which is a random variable on $\left(\widetilde{\Omega}^{\otimes N}, \widetilde{\mathcal{A}}^{\otimes N}, \widetilde{\mathrm{P}}^{\otimes N}\right)$ of law $\nu^{\otimes N}$. The expectancy against $\mathrm{P}$ will be denoted by $\mathbb{E}($.$) .$

As a bounded perturbation of $-\frac{\mathrm{d}^{2}}{\mathrm{~d} x^{2}} \otimes I_{\mathrm{N}}$, the operator $H_{\ell}(\omega)$ is self-adjoint on the Sobolev space $H^{2}(\mathbb{R}) \otimes \mathbb{R}^{N}$ and thus, for every $\omega \in \Omega$, the spectrum of $H_{\ell}(\omega)$, $\sigma\left(H_{\ell}(\omega)\right)$, is included in $\mathbb{R}$. Moreover, because of the periodicity in law of the random potential of $H_{\ell}(\omega)$, the family $\left\{H_{\ell}(\omega)\right\}_{\omega \in \Omega}$ is $\ell \mathbb{Z}$-ergodic. Thus, there exists $\Sigma \subset \mathbb{R}$ such that, for P-almost every $\omega \in \Omega, \Sigma=\sigma\left(H_{\ell}(\omega)\right)$. There also exist $\Sigma_{\mathrm{pp}}$, $\Sigma_{\mathrm{ac}}$ and $\Sigma_{\mathrm{sc}}$, subsets of $\mathbb{R}$, such that, for P-almost every $\omega \in \Omega, \Sigma_{\mathrm{pp}}=\sigma_{\mathrm{pp}}\left(H_{\ell}(\omega)\right)$, 
$\Sigma_{\mathrm{ac}}=\sigma_{\mathrm{ac}}\left(H_{\ell}(\omega)\right)$ and $\Sigma_{\mathrm{sc}}=\sigma_{\mathrm{sc}}\left(H_{\ell}(\omega)\right)$, respectively the pure point, absolutely continuous and singular continuous spectrum of $H_{\ell}(\omega)$.

Our main result will be about localization properties of $H_{\ell}(\omega)$. Before stating it, we give the definitions of both exponential localization and dynamical localization for $H_{\ell}(\omega)$. We denote by $E_{\omega}($.$) the spectral projection of the self-adjoint operator$ $H_{\ell}(\omega)$ and the $L^{2}$-norm is written as \|\| .

Definition 1. Let $I \subset \mathbb{R}$ be an open interval. We say that :

(i) $H_{\ell}(\omega)$ exhibits exponential localization (EL) in I, if it has pure point spectrum in $I$ (i.e., $\Sigma \cap I=\Sigma_{\mathrm{pp}} \cap I$ and $\Sigma_{\mathrm{ac}} \cap I=\Sigma_{\mathrm{sc}} \cap I=\emptyset$ ) and, for P-almost every $\omega \in \Omega$, the eigenfunctions of $H_{\ell}(\omega)$ with eigenvalues in $I$ decay exponentially in the $L^{2}$-sense (i.e., there exist $C$ and $m>0$ such that $\left\|\mathbf{1}_{[x-\ell, x+\ell]} \psi\right\| \leq C \mathrm{e}^{-m|x|}$ for $\psi$ an eigenfunction of $\left.H_{\ell}(\omega)\right)$;

(ii) $H_{\ell}(\omega)$ exhibits strong dynamical localization $(S D L)$ in $I$, if $\Sigma \cap I \neq \emptyset$ and, for each compact interval $\tilde{I} \subset I$ and $\psi \in L^{2}(\mathbb{R}) \otimes \mathbb{R}^{N}$ with compact support, we have,

$$
\forall n \geq 0, \mathbb{E}\left(\sup _{t \in \mathbb{R}}\left\|\left(\sqrt{1+|x|^{2}}\right)^{\frac{n}{2}} E_{\omega}(\tilde{I}) \mathrm{e}^{-\mathrm{i} t H_{\ell}(\omega)} \psi\right\|^{2}\right)<\infty .
$$

Before stating our main results, we need to introduce some more notations. Let $\mathrm{Sp}_{\mathrm{N}}(\mathbb{R})$ denote the group of $2 \mathrm{~N} \times 2 \mathrm{~N}$ real symplectic matrices. It is the subgroup of $\mathrm{GL}_{2 \mathrm{~N}}(\mathbb{R})$ of matrices $M$ satisfying

$$
{ }^{t} M J M=J,
$$

where $J$ is the matrix of order $2 N$ defined by $J=\left(\begin{array}{cc}0 & -I_{N} \\ I_{N} & 0\end{array}\right)$. Let $\mathcal{O}$ be the neighborhood of $I_{2 \mathrm{~N}}$ in $\operatorname{Sp}_{\mathrm{N}}(\mathbb{R})$ given by Theorem 2 applied to $G=\mathrm{Sp}_{\mathrm{N}}(\mathbb{R})$.

We set :

$$
\mathrm{d}_{\log \mathcal{O}}=\max \{R>0 \mid B(0, R) \subset \log \mathcal{O}\},
$$

where $B(0, R)$ is the open ball, centered on 0 and of radius $R>0$, for the topology induced on the Lie algebra $\mathfrak{s p}_{\mathrm{N}}(\mathbb{R})$ of $\operatorname{Sp}_{\mathrm{N}}(\mathbb{R})$ by the matrix norm induced by the euclidean norm on $R^{2 N}$.

For $\omega^{(0)}=\left(\omega_{1}^{(0)}, \ldots, \omega_{N}^{(0)}\right) \in\{0,1\}^{N}$, let

$$
M_{\omega(0)}(0, V)=V+\operatorname{diag}\left(c_{1} \omega_{1}^{(0)}, \ldots, c_{N} \omega_{N}^{(0)}\right) .
$$

As $M_{\omega^{(0)}}(0, V) \in \mathrm{S}_{\mathrm{N}}(\mathbb{R})$, it has $\lambda_{1}^{\omega^{(0)}}, \ldots, \lambda_{N}^{\omega^{(0)}}$ as real eigenvalues. We set,

$$
\lambda_{\min }=\min _{\omega^{(0)} \in\{0,1\}^{N}} \min _{1 \leq i \leq N} \lambda_{i}^{\omega^{(0)}}, \quad \lambda_{\max }=\max _{\omega^{(0)} \in\{0,1\}^{N}} \max _{1 \leq i \leq N} \lambda_{i}^{\omega^{(0)}}
$$

and $\delta=\frac{\lambda_{\max }-\lambda_{\min }}{2}$. We also set

$$
\ell_{C}:=\ell_{C}(N, V)=\min \left(1, \frac{\mathrm{d}_{\log \mathcal{O}}}{\delta}\right)
$$

and, for every $\ell \in\left(0, \ell_{C}\right)$,

$$
I(N, V, \ell)=\left[\lambda_{\max }-\frac{\mathrm{d}_{\log \mathcal{O}}}{\ell}, \lambda_{\min }+\frac{\mathrm{d}_{\log \mathcal{O}}}{\ell}\right] .
$$

We remark that, as $\ell$ tends to $0^{+}, I(N, V, \ell)$ tends to the whole real line. We can now state our main result. 
Theorem 1. For almost every $V \in \mathrm{S}_{\mathrm{N}}(\mathbb{R})$, there exists a finite set $\mathcal{S}_{\mathrm{V}} \subset \mathbb{R}$ such that, for every $\ell \in\left(0, \ell_{C}\right)$, if $I \subset I(N, V, \ell) \backslash \mathcal{S}_{\mathrm{V}}$ is an open interval with $\Sigma \cap I \neq \emptyset$, then $H_{\ell}(\omega)$ exhibits $(E L)$ and $(S D L)$ on $I$.

Here, "almost every" is considered according to the Lebesgue measure on $\mathrm{S}_{\mathrm{N}}(\mathbb{R})$ identified to $\mathbb{R}^{\frac{N(N+1)}{2}}$. We also remark that, as $I(N, V, \ell)$ tends to $\mathbb{R}$ when $\ell$ tends to $0^{+}$, taking $\ell \in\left(0, \ell_{C}\right)$ small enough ensure that we can always find a non-trivial open interval $I \subset I(N, V, \ell) \backslash \mathcal{S}_{\mathrm{V}}$ such that $\Sigma \cap I \neq \emptyset$.

This theorem will follow from the next proposition. For $E \in \mathbb{R}$, let $G(E)$ be the Fürstenberg group associated to $H_{\ell}(\omega)$ (see Definition (3).

Proposition 1. For almost every $V \in \mathrm{S}_{\mathrm{N}}(\mathbb{R})$, there exists a finite set $\mathcal{S}_{\mathrm{V}} \subset \mathbb{R}$ such that, for every $\ell \in\left(0, \ell_{C}\right)$,

$$
\forall E \in I(N, V, \ell) \backslash \mathcal{S}_{\mathrm{V}}, G(E)=\operatorname{Sp}_{\mathrm{N}}(\mathbb{R}) .
$$

In particular, Proposition 1 will imply the separability of the Lyapunov exponents of $H_{\ell}(\omega)$ (see Definition 2) and the absence of absolutely continuous spectrum in $I(N, V, \ell)$, for $\ell \in\left(0, \ell_{C}\right)$.

Corollary 1. For almost every $V \in \mathrm{S}_{\mathrm{N}}(\mathbb{R})$, there exists a finite set $\mathcal{S}_{\mathrm{V}} \subset \mathbb{R}$ such that, for every $\ell \in\left(0, \ell_{C}\right)$, the $N$ positive Lyapunov exponents of $H_{\ell}(\omega)$, $\gamma_{1}(E), \ldots, \gamma_{N}(E)$, verify:

$$
\forall E \in I(N, V, \ell) \backslash \mathcal{S}_{\mathrm{V}}, \quad \gamma_{1}(E)>\cdots>\gamma_{N}(E)>0 .
$$

Therefore, $H_{\ell}(\omega)$ has no absolutely continuous spectrum in $I(N, V, \ell)$, i.e., for every $\ell \in\left(0, \ell_{C}\right), \Sigma_{\mathrm{ac}} \cap I(N, V, \ell)=\emptyset$.

It is already known in the scalar-valued case (corresponding here to $N=1$ ) that, away from a discrete set $\mathcal{S}$ of critical energies, there is exponential localization and strong dynamical localization on every compact interval $I \subset \mathbb{R} \backslash \mathcal{S}$ with $\Sigma \cap I \neq \emptyset$ (see [6]). But, in dimension $d$ higher than 2, the question of the localization remains mostly open for Anderson-Bernoulli models. Such an Anderson-Bernoulli model is given by a family of random operators of the form

$$
H(\omega)=-\Delta_{d}+\sum_{n \in \mathbb{Z}^{d}} \omega_{n} V(x-n),
$$

acting on $L^{2}\left(\mathbb{R}^{d}\right) \otimes \mathbb{R}$, where $V$ is supported in $[0,1]^{d}$ and the $\omega_{n}$ are i.i.d. Bernoulli random variables. Since [4, it is known that there is exponential localization at the bottom of the almost sure spectrum of $H(\omega)$. In dimension $d \geq 3$, it is commonly conjectured that for high energies, there exist extended states, as for dimension $d=2$ it is conjectured that there is localization at every energies, except maybe those in a discrete set.

To tackle the question of localization for $d=2$, we can start by looking at a slightly simpler model, a continuous strip $\mathbb{R} \times[0,1]$ in $\mathbb{R}^{2}$. This model is given by the restriction $H_{\mathrm{cs}}(\omega)$ of $H(\omega)$ to $L^{2}(\mathbb{R} \times[0,1])$, with Dirichlet boundary conditions on $\mathbb{R} \times\{0\}$ and $\mathbb{R} \times\{1\}$. This model can be used to study transport properties of nanoconductors so it is also of physical interest. The question of the localization at all energies for $H_{\mathrm{cS}}(\omega)$ present difficulties of the same level as for $H(\omega)$, mostly 
due to the PDE's nature of the problem in both cases. But, for $H_{\mathrm{cs}}(\omega)$, we have a possible approach by operating a discretization in the bounded direction of the strip. This can be performed by first applying discrete Fourier transform in the second variable corresponding to the bounded direction, which leads to a quasi onedimensional model with an infinite size matrix for potential. Then, by applying a cut-off in the space of Fourier frequencies, we obtain a quasi one-dimensional model with a matrix of finite order $N$ for potential, acting on $L^{2}(\mathbb{R}) \otimes \mathbb{R}^{N}$, with $N \geq 1$ an integer. It turns the nature of the initial PDE's problem to an ODE's one, which allows to use formalism such as transfer matrices and Lyapunov exponents. The model (11) we are looking at here is not exactly the one obtained by this discretization procedure, but the understanding of localization properties for (11) should lead us to the same understanding for the discretize operator obtained from $H_{\mathrm{cs}}(\omega)$.

We finish this introduction by giving the outline of the article. In Section 2 , we present the formalism of transfer matrices and compute them for $H_{\ell}(\omega)$. We also define the Lyapunov exponents and the Fürstenberg group associated to $H_{\ell}(\omega)$. In Section 3, we study the Lie algebra generated by the matrices $X_{\omega(0)}(E, V)$ defined at (15). In this section we also prove the genericity argument and we construct the finite set $\mathcal{S}_{\mathrm{V}}$ of Theorem 1, Proposition 1 and Corollary 1, This genericity argument is mostly based upon algebraic geometry considerations and the Lebesgue measure of affine algebraic manifolds. In Section 4, we prove Proposition 1 and Corollary 1 and we explicitely construct $\ell_{C}$ and $I(N, V, \ell)$ for $\ell \in\left(0, \ell_{C}\right)$. The proofs of this section are based upon a general result on Lie groups due to Breuillard and Gelander (see Theorem 22). In Section [5, we recall localization results of [3] and we deduce from them the proof of Theorem 1. Finally, in Section [6 we state a result of existence and regularity of the integrated density of states associated to $H_{\ell}(\omega)$.

The general idea of the proof of Theorem 1 can be briefly sketched. First we change the initial spectral and dynamical problem of the localization into a topological problem on proving that a Lie group with a finite number of generators is dense in the real symplectic group $\operatorname{Sp}_{N}(\mathbb{R})$, which is the statement of Proposition 1. Then, we use the general criterion on Lie groups of Breuillard and Gelander to transform this topological problem into a purely algebraic problem on generating the Lie algebra $\mathfrak{s p}_{\mathrm{N}}(\mathbb{R})$. The algebraic nature of the objects we are considering at this last step allows us to prove a generic result on $V$ and the finiteness of the set $\mathcal{S}_{\mathrm{V}}$ of critical energies.

\section{Transfer matrices and the Fürstenberg group}

Let $E \in \mathbb{R}$. We want to understand the exponential asymptotic behaviour of a solution $u: \mathbb{R} \rightarrow \mathbb{R}^{N}$ of the second order differential system

$$
H_{\ell}(\omega) u=E u .
$$

For this, we transform (8) into an Hamiltonian differential system of order 1 and we introduce the transfer matrix $T_{\omega^{(n)}}(E)$ of $H_{\ell}(\omega)$ from $\ell n$ to $\ell(n+1)$ which maps a solution $\left(u, u^{\prime}\right)$ of the order 1 system at time $\ell n$ to the solution at time $\ell(n+1)$. The transfer matrix $T_{\omega(n)}(E)$ is therefore defined by the relation

$$
\forall n \in \mathbb{Z},\left(\begin{array}{c}
u(\ell(n+1)) \\
u^{\prime}(\ell(n+1))
\end{array}\right)=T_{\omega^{(n)}}(E)\left(\begin{array}{c}
u(\ell n) \\
u^{\prime}(\ell n)
\end{array}\right) .
$$


As the system of order 1 is Hamiltonian, the transfer matrix $T_{\omega^{(n)}}(E)$ lies into the symplectic group $\operatorname{Sp}_{\mathrm{N}}(\mathbb{R})$. The sequence $\left(T_{\omega^{(n)}}(E)\right)_{n \in \mathbb{Z}}$ is also a sequence of i.i.d. symplectic matrices because of the i.i.d. character of the $\omega_{i}^{(n)}$ 's and the non-overlapping of these random variables. By iterating the relation (9) we get the asymptotic behaviour of $\left(u, u^{\prime}\right)$. To get the exponential asymptotic behaviour of $\left(u, u^{\prime}\right)$ we can define the exponential growth (or decay) exponents of the product of random matrices $T_{\omega(n-1)}(E) \ldots T_{\omega(0)}(E)$.

Definition 2. Let $E \in \mathbb{R}$. The Lyapunov exponents $\gamma_{1}(E), \ldots, \gamma_{2 N}(E)$, associated to the sequence $\left(T_{\omega(n)}(E)\right)_{n \in \mathbb{Z}}$, are defined inductively by

$$
\sum_{i=1}^{p} \gamma_{i}(E)=\lim _{n \rightarrow \infty} \frac{1}{n} \mathbb{E}\left(\log \left\|\wedge^{p}\left(T_{\omega(n-1)}(E) \ldots T_{\omega(0)}(E)\right)\right\|\right),
$$

for every $p \in\{1, \ldots, 2 N\}$.

Here, $\wedge^{p} M$ denotes the $p$ th exterior power of the matrix $M$, acting on the $p$ th exterior power of $\mathbb{R}^{2 N}$. One has $\gamma_{1}(E) \geq \ldots \geq \gamma_{2 N}(E)$. Moreover, due to the symplecticity of the random matrices $T_{\omega(n)}(E)$, we have the symmetry property $\gamma_{2 N-i+1}=-\gamma_{i}$, for every $i \in\{1, \ldots, N\}$. Thus, we will only have to study the $N$ first Lyapunov exponents to obtain Corollary 1. To prove the separability of the Lyapunov exponents, we introduce the group which contains all the different products of transfer matrices, the so-called Fürstenberg group.

Definition 3. For every $E \in \mathbb{R}$, the Fürstenberg group of $H_{\ell}(\omega)$ is defined by

$$
G(E)=\overline{\operatorname{supp} \mu_{E}>},
$$

where $\mu_{E}$ is the common distribution of the $T_{\omega(n)}(E)$ and the closure is taken for the usual topology in $\mathrm{Sp}_{\mathrm{N}}(\mathbb{R})$.

As the $T_{\omega^{(n)}}(E)$ are i.i.d., $\mu_{E}=\left(T_{\omega^{(0)}}(E)\right)_{*} \nu^{\otimes N}$ and we have the internal description of $G(E)$ :

$$
\forall E \in \mathbb{R}, G(E)=\overline{\left\langle T_{\omega^{(0)}}(E)\right| \omega^{(0)} \in \operatorname{supp} \nu^{\otimes N}>} .
$$

As $\{0,1\} \subset \operatorname{supp} \nu$, we also have

$$
\overline{<T_{\omega(0)}(E) \mid \omega^{(0)} \in\{0,1\}^{N}>} \subset G(E) .
$$

We will denote be $G_{\{0,1\}}(E)$ the subgroup of $G(E)$ with $2^{N}$ generators :

$$
G_{\{0,1\}}(E)=<T_{\omega(0)}(E) \mid \omega^{(0)} \in\{0,1\}^{N}>.
$$

In Section 4, we will prove that, for almost every $V \in \mathrm{S}_{\mathrm{N}}(\mathbb{R})$ and for all $E \in \mathbb{R}$ except those in a finite set, $G_{\{0,1\}}(E)$ is dense in $\operatorname{Sp}_{\mathrm{N}}(\mathbb{R})$.

We finish this section by giving the explicit form of the transfer matrices $T_{\omega^{(n)}}(E)$. Let $V \in \mathrm{S}_{\mathrm{N}}(\mathbb{R}), E \in \mathbb{R}, n \in \mathbb{Z}$ and $\omega^{(n)} \in \tilde{\Omega}^{\otimes N}$. We set :

$$
M_{\omega^{(n)}}(E, V)=V+\operatorname{diag}\left(c_{1} \omega_{1}^{(n)}, \ldots, c_{N} \omega_{N}^{(n)}\right)-E I_{\mathrm{N}} .
$$

Then, we set the following matrix of the Lie algebra $\mathfrak{s p}_{\mathrm{N}}(\mathbb{R})$,

$$
X_{\omega^{(n)}}(E, V)=\left(\begin{array}{cc}
0 & I_{\mathrm{N}} \\
M_{\omega^{(n)}}(E, V) & 0
\end{array}\right) \in \mathfrak{s p}_{\mathrm{N}}(\mathbb{R}) \subset \mathcal{M}_{2 \mathrm{~N}}(\mathbb{R}) .
$$


By solving the constant coefficients system (8) on $[\ell n, \ell(n+1)]$, we have :

$$
\forall \ell>0, \forall n \in \mathbb{Z}, \forall V \in \mathrm{S}_{\mathrm{N}}(\mathbb{R}), \forall E \in \mathbb{R}, T_{\omega^{(n)}}(E)=\exp \left(\ell X_{\omega^{(n)}}(E, V)\right) .
$$

It is important here to notice that $T_{\omega^{(n)}}(E)$ is the exponential of a matrix, as it will be crucial to be able to apply Theorem 2 to the subgroup $G_{\{0,1\}}(E)$.

\section{The Lie algebra generated By $\left\{X_{\omega^{(0)}}(E, V) \mid \omega^{(0)} \in\{0,1\}^{N}\right\}$}

In this section we will present in details the proof of the genericity argument needed to prove Proposition 1 and Theorem 1 . We start by looking at the geometry of the set of $k$-uples in $\mathfrak{s p}_{N}(\mathbb{R})$ which do not generates $\mathfrak{s p}_{N}(\mathbb{R})$ in the sense of Lie algebras.

Lemma 1. Let $k \in \mathbb{N}^{*}$ and

$$
\mathcal{V}_{k}=\left\{\left(X_{1}, \ldots, X_{k}\right) \in\left(\mathfrak{s p}_{\mathrm{N}}(\mathbb{R})\right)^{k} \mid\left(X_{1}, \ldots, X_{k}\right) \text { does not generate } \mathfrak{s p}_{\mathrm{N}}(\mathbb{R})\right\} .
$$

Then, there exist $Q_{r_{1}}, \ldots, Q_{r_{k}} \in \mathbb{R}\left[\left(\mathfrak{s p}_{\mathrm{N}}(\mathbb{R})\right)^{k}\right]$ such that :

$$
\mathcal{V}_{k}=\left\{\left(X_{1}, \ldots, X_{k}\right) \in\left(\mathfrak{s p}_{\mathrm{N}}(\mathbb{R})\right)^{k} \mid Q_{r_{1}}\left(X_{1}, \ldots, X_{k}\right)=0, \ldots, Q_{r_{k}}\left(X_{1}, \ldots, X_{k}\right)=0\right\} .
$$

Thus, $\mathcal{V}_{k}$ is the affine algebraic manifold of $\left\{Q_{r_{1}}, \ldots, Q_{r_{k}}\right\}$ which will be denoted by $V\left(\left\{Q_{r_{1}}, \ldots, Q_{r_{k}}\right\}\right)$. We will also use the identification :

$$
\mathbb{R}\left[\left(\mathfrak{s p}_{\mathrm{N}}(\mathbb{R})\right)^{k}\right] \simeq \mathbb{R}\left[T_{1}, \ldots, T_{k\left(2 N^{2}+N\right)}\right] .
$$

Proof. Let $\left(X_{1}, \ldots, X_{k}\right) \in\left(\mathfrak{s p}_{\mathrm{N}}(\mathbb{R})\right)^{k}$ and $\operatorname{Lie}\left\{X_{1}, \ldots, X_{k}\right\}$ be the Lie algebra generated by $X_{1}, \ldots, X_{k}$. If we denote by $\left\{Y_{1}, \ldots, Y_{l}, \ldots\right\}$ the countable set of all the successives brackets constructed from $\left\{X_{1}, \ldots, X_{k}\right\}$, we have

$$
\operatorname{Lie}\left\{X_{1}, \ldots, X_{k}\right\}=\operatorname{span}\left(\left\{Y_{1}, \ldots, Y_{l}, \ldots\right\}\right),
$$

the vector space spanned by $\left\{Y_{1}, \ldots, Y_{l}, \ldots\right\}$. Then we have :

$$
\operatorname{Lie}\left\{X_{1}, \ldots, X_{k}\right\} \neq \mathfrak{s p}_{N}(\mathbb{R}) \Leftrightarrow \operatorname{rk}\left(\left\{Y_{1}, \ldots, Y_{l}, \ldots\right\}\right)<2 N^{2}+N,
$$

as $\operatorname{dim} \mathfrak{s p}_{\mathrm{N}}(\mathbb{R})=2 N^{2}+N$. At each $Y_{l} \in \mathfrak{s p}_{\mathrm{N}}(\mathbb{R})$ we associate $\tilde{Y}_{l} \in \mathbb{R}^{2 N^{2}+N}$ whose coefficients are those which define the matrix $Y_{l}$. The coefficients of $\tilde{Y}_{l}$ are polynomial in the $k\left(2 N^{2}+N\right)$ coefficients which define the matrices $X_{1}, \ldots X_{k}$. For $m \in\left(\mathbb{N}^{*}\right)^{2 N^{2}+N}$, we set

$$
Q_{m}\left(X_{1}, \ldots, X_{k}\right)=\operatorname{det}\left(\tilde{Y}_{m_{1}}, \ldots, \tilde{Y}_{m_{2 N^{2}+N}}\right) \in \mathbb{R}\left[\left(\mathfrak{s p}_{N}(\mathbb{R})\right)^{k}\right]
$$

Then,

(23) $\operatorname{rk}\left(\left\{Y_{1}, \ldots, Y_{l}, \ldots\right\}\right)<2 N^{2}+N \Leftrightarrow \forall m \in\left(\mathbb{N}^{*}\right)^{2 N^{2}+N}, Q_{m}\left(X_{1}, \ldots X_{k}\right)=0$.

Thus,

$$
\mathcal{V}_{k}=\bigcap_{m \in\left(\mathbb{N}^{*}\right)^{2 N^{2}+N}}\left\{\left(X_{1}, \ldots, X_{k}\right) \in\left(\mathfrak{s p}_{\mathrm{N}}(\mathbb{R})\right)^{k} \mid Q_{m}\left(X_{1}, \ldots, X_{k}\right)=0\right\} .
$$

With the definition of the affine algebraic manifold, we can rewrite (24) as :

$$
\mathcal{V}_{k}=V\left(\left\{Q_{m} \mid m \in\left(\mathbb{N}^{*}\right)^{2 N^{2}+N}\right\}\right) .
$$


But, if $I\left(\left\{Q_{m} \mid m \in\left(\mathbb{N}^{*}\right)^{2 N^{2}+N}\right\}\right)$ denote the ideal generated by the family $\left\{Q_{m} \mid m \in\left(\mathbb{N}^{*}\right)^{2 N^{2}+N}\right\}$, we have :

$$
V\left(\left\{Q_{m} \mid m \in\left(\mathbb{N}^{*}\right)^{2 N^{2}+N}\right\}\right)=V\left(I\left(\left\{Q_{m} \mid m \in\left(\mathbb{N}^{*}\right)^{2 N^{2}+N}\right\}\right)\right) .
$$

As the ring $\mathbb{R}\left[T_{1}, \ldots, T_{k\left(2 N^{2}+N\right)}\right]$ is Noetherian, $I\left(\left\{Q_{m} \mid m \in\left(\mathbb{N}^{*}\right)^{2 N^{2}+N}\right\}\right)$ is of finite type, i.e. there exist $r_{1}, \ldots, r_{k} \in\left(\mathbb{N}^{*}\right)^{2 N^{2}+N}$ such that,

$$
I\left(\left\{Q_{m} \mid m \in\left(\mathbb{N}^{*}\right)^{2 N^{2}+N}\right\}\right)=I\left(\left\{Q_{r_{1}}, \ldots, Q_{r_{k}}\right\}\right) .
$$

Finally,

$$
\mathcal{V}_{k}=V\left(I\left(\left\{Q_{r_{1}}, \ldots, Q_{r_{k}}\right\}\right)\right)=V\left(\left\{Q_{r_{1}}, \ldots, Q_{r_{k}}\right\}\right) .
$$

For $E \in \mathbb{R}$ and $V \in \mathrm{S}_{\mathrm{N}}(\mathbb{R})$, we will reindex the family $\left\{X_{\omega^{(0)}}(E, V)\right\}_{\omega^{(0)} \in\{0,1\}^{N}}$ as $\left(X_{1}(E, V), \ldots, X_{2^{N}}(E, V)\right)$. Let $E \in \mathbb{R}$ be fixed and let

$$
\mathcal{V}_{(E)}=\left\{V \in \mathrm{S}_{\mathrm{N}}(\mathbb{R}) \mid\left(X_{1}(E, V), \ldots, X_{2^{N}}(E, V)\right) \text { does not generate } \mathfrak{s p}_{\mathrm{N}}(\mathbb{R})\right\} .
$$

Lemma 2. We have, $\operatorname{Leb}_{\frac{N(N+1)}{2}}\left(\mathcal{V}_{(E)}\right)=0$.

Proof. Let

$$
\begin{aligned}
f_{E}: \mathrm{S}_{\mathrm{N}}(\mathbb{R}) & \rightarrow\left(\mathfrak{s p}_{\mathrm{N}}(\mathbb{R})\right)^{2^{N}} \\
V & \mapsto\left(X_{1}(E, V), \ldots, X_{2^{N}}(E, V)\right)
\end{aligned}
$$

Then $f_{E}$ is polynomial in the $\frac{N(N+1)}{2}$ coefficients which define $V$. Indeed, we can identify $\mathrm{S}_{\mathrm{N}}(\mathbb{R}) \simeq \mathbb{R}^{\frac{N(N+1)}{2}}$ and $\left(\mathfrak{s p}_{\mathrm{N}}(\mathbb{R})\right)^{2^{N}} \simeq \mathbb{R}^{2^{N}\left(2 N^{2}+N\right)}$ and, after this identification, $f_{E}$ has each of its $2^{N}\left(2 N^{2}+N\right)$ components polynomial in $\mathbb{R}\left[T_{1}, \ldots, T_{\frac{N(N+1)}{2}}\right]$. We have :

$$
\mathcal{V}_{(E)}=f_{E}^{-1}\left(\mathcal{V}_{2^{N}}\right)
$$

Then, by Lemma 1, $V \in \mathcal{V}_{(E)}$ if and only if

$$
Q_{r_{1}}\left(X_{1}(E, V), \ldots, X_{2^{N}}(E, V)\right)=0, \ldots, Q_{r_{2^{N}}}\left(X_{1}(E, V), \ldots, X_{2^{N}}(E, V)\right)=0,
$$

which can be rewrite

$$
V \in \mathcal{V}_{(E)} \Leftrightarrow\left(Q_{r_{1}} \circ f_{E}\right)(V)=0, \ldots,\left(Q_{r_{2 N}} \circ f_{E}\right)(V)=0 .
$$

But, we can prove that, if $V_{0}$ is the tridiagonal matrix with zeros on the diagonal and all coefficients on its upper and lower diagonals equal to 1 , then, for any $E \in \mathbb{R}$, $V_{0} \notin \mathcal{V}_{(E)}$ (see [3, Lemma 3]). Thus, there exists $i_{0} \in\left\{r_{1}, \ldots, r_{2^{N}}\right\}$ such that $\left(Q_{i_{0}} \circ f_{E}\right)\left(V_{0}\right) \neq 0$ and, as the function $Q_{i_{0}} \circ f_{E}$ is polynomial and do not vanish identically,

$$
\left.\operatorname{Leb}_{\frac{N(N+1)}{2}}\left(\left\{V \in \mathrm{S}_{N}(\mathbb{R}) \mid\left(Q_{i_{0}} \circ f_{E}\right)(V)=0\right)\right\}\right)=0,
$$

and, by inclusion,

$$
\operatorname{Leb}_{\frac{N(N+1)}{2}}\left(\mathcal{V}_{(E)}\right)=0
$$


Finally, we can introduce the set :

$$
\mathcal{V}=\bigcap_{E \in \mathbb{R}} \mathcal{V}_{(E)}
$$

$=\left\{V \in \mathrm{S}_{\mathrm{N}}(\mathbb{R}) \mid \forall E \in \mathbb{R},\left(X_{1}(E, V), \ldots, X_{2^{N}}(E, V)\right)\right.$ does not generate $\left.\mathfrak{s p}_{\mathrm{N}}(\mathbb{R})\right\}$. Then, by Lemma 2 and by inclusion, we have :

$$
\operatorname{Leb}_{\frac{N(N+1)}{2}}(\mathcal{V})=0
$$

Now we can prove the last result of this section.

Lemma 3. For any $V \in \mathrm{S}_{\mathrm{N}}(\mathbb{R}) \backslash \mathcal{V}$, there exists a finite set $\mathcal{S}_{\mathrm{V}} \subset \mathbb{R}$ such that :

$$
\forall E \in \mathbb{R} \backslash \mathcal{S}_{\mathrm{V}},\left(X_{1}(E, V), \ldots, X_{2^{N}}(E, V)\right) \text { generates } \mathfrak{s p}_{\mathrm{N}}(\mathbb{R}) .
$$

Proof. Let $V \in \mathrm{S}_{\mathrm{N}}(\mathbb{R}) \backslash \mathcal{V}$. Then, there exists $E_{0} \in \mathbb{R}$ such that the family $\left(X_{1}\left(E_{0}, V\right), \ldots, X_{2^{N}}\left(E_{0}, V\right)\right)$ generates $\mathfrak{s p}_{\mathrm{N}}(\mathbb{R})$. Thus, there exists $i_{0} \in\left\{r_{1}, \ldots, r_{2^{N}}\right\}$ such that $\left(Q_{i_{0}} \circ f\right)\left(E_{0}, V\right) \neq 0$, where

$$
f: \begin{array}{cl}
\mathbb{R} \times \mathrm{S}_{\mathrm{N}}(\mathbb{R}) & \rightarrow\left(\mathfrak{s p}_{\mathrm{N}}(\mathbb{R})\right)^{2^{N}} \\
(E, V) & \mapsto\left(X_{1}(E, V), \ldots, X_{2^{N}}(E, V)\right)
\end{array}
$$

But, for $V$ fixed, $E \mapsto\left(Q_{i_{0}} \circ f\right)(E, V)$ is polynomial and, as it is not identically vanishing, it has only a finite set $\mathcal{S}_{\mathrm{V}}$ of roots. Thus, we have :

$$
\forall E \in \mathbb{R} \backslash \mathcal{S}_{\mathrm{V}},\left(Q_{i_{0}} \circ f\right)(E, V) \neq 0,
$$

which is equivalent to :

$$
\forall E \in \mathbb{R} \backslash \mathcal{S}_{\mathrm{V}},\left(X_{1}(E, V), \ldots, X_{2^{N}}(E, V)\right) \notin \mathcal{V}_{2^{N}} .
$$

With this Lemma 3 we are now able to prove Proposition 1

\section{Proof of Proposition 1 and Corollary 1}

The proof of Proposition 1 is based upon a general criterion of density in semisimple Lie groups due to Breuillard and Gelander.

Theorem 2 ([5], Theorem 2.1). Let $G$ be a real, connected, semisimple Lie group, whose Lie algebra is $\mathfrak{g}$. Then, there is a neighborhood $\mathcal{O}$ of 1 in $G$, on which $\log =\exp ^{-1}$ is a well defined diffeomorphism, such that $g_{1}, \ldots, g_{m} \in \mathcal{O}$ generate a dense subgroup whenever $\log g_{1}, \ldots, \log g_{m}$ generate $\mathfrak{g}$.

This criterion, applied to $G=\mathrm{Sp}_{\mathrm{N}}(\mathbb{R})$, gives us the outline of the proof of Proposition 1:

(i) We construct $\ell_{C}$ and $I(N, V, \ell)$ such that, for $\ell \in\left(0, \ell_{C}\right)$ and $E \in I(N, V, \ell)$, $T_{\omega(0)}(E) \in \mathcal{O}$, for every $\omega^{(0)} \in\{0,1\}^{N}$.

(ii) We compute $\log T_{\omega(0)}(E)$.

(iii) We justify that $\operatorname{Lie}\left\{\log T_{\omega(0)}(E) \mid \omega^{(0)} \in\{0,1\}^{N}\right\}=\mathfrak{s p}_{\mathrm{N}}(\mathbb{R})$ for $V \in$ $\mathrm{S}_{\mathrm{N}}(\mathbb{R}) \backslash \mathcal{V}$ and $E \in I(N, V, \ell) \backslash \mathcal{S}_{\mathrm{V}}$.

(iv) We deduce that $G_{\{0,1\}}(E)$ is dense for the usual topology in $\operatorname{Sp}_{N}(\mathbb{R})$, for $E \in I(N, V, \ell) \backslash \mathcal{S}_{\mathrm{V}}$. 
Proof. We fix $V \in \mathrm{S}_{\mathrm{N}}(\mathbb{R}) \backslash \mathcal{V}$. We start be constructing $\ell_{C}$ and, for $\ell \in\left(0, \ell_{C}\right)$, the interval $I(N, V, \ell)$ as given in (4) and (5). Now, let $\lambda_{1}^{\omega^{(0)}}, \ldots, \lambda_{N}^{\omega^{(0)}}$ be the real eigenvalues of $M_{\omega^{(0)}}(0, V)$ (see (14)). Then, the eigenvalues of $X_{\omega^{(0)}}(E, V)^{t} X_{\omega^{(0)}}(E, V)$ are $1,\left(\lambda_{1}^{\omega^{(0)}}-E\right)^{2}, \ldots,\left(\lambda_{N}^{\omega^{(0)}}-E\right)^{2}$, thus :

$$
\left\|X_{\omega^{(0)}}(E, V)\right\|=\max \left(1, \max _{1 \leq i \leq N}\left|\lambda_{i}^{\omega^{(0)}}-E\right|\right),
$$

where \|\| is the matrix norm associated to the euclidian norm on $\mathbb{R}^{2 N}$.

Let $\mathcal{O}$ be the neighborhood of the identity given by Theorem 2 applied to the group $G=\operatorname{Sp}_{\mathrm{N}}(\mathbb{R})$. Then, for $\mathrm{d}_{\log \mathcal{O}}$ as defined in (2), we take $\ell \leq \mathrm{d}_{\log \mathcal{O}}$ and we set $r_{\ell}=\frac{1}{\ell} d_{\log \mathcal{O}} \geq 1$. If we set

$$
I(N, V, \ell)=\left\{E \in \mathbb{R} \mid \max \left(1, \max _{\omega(0) \in\{0,1\}^{N}} \max _{1 \leq i \leq N}\left|\lambda_{i}^{\omega^{(0)}}-E\right|\right) \leq r_{\ell}\right\},
$$

as $r_{\ell} \geq 1$,

$$
I(N, V, \ell)=\bigcap_{\omega^{(0)} \in\{0,1\}^{N}} \bigcap_{1 \leq i \leq N}\left[\lambda_{i}^{\omega^{(0)}}-r_{\ell}, \lambda_{i}^{\omega^{(0)}}+r_{\ell}\right] .
$$

Let $\lambda_{\min }, \lambda_{\max }$ and $\delta$ be as in (3). If $\delta<r_{\ell}$ then $I(N, V, \ell) \neq \emptyset$ and we have

$$
I(N, V, \ell)=\left[\lambda_{\max }-r_{\ell}, \lambda_{\min }+r_{\ell}\right],
$$

which is the definition we took in (5). This interval is centered in $\frac{\lambda_{\min }+\lambda_{\max }}{2}$ and is of length $2 r_{\ell}-2 \delta>0$, which tends to $+\infty$ when $\ell$ tends to $0^{+}$. We also note that $\lambda_{\min }, \lambda_{\max }$ and $\mathrm{d}_{\log \mathcal{O}}$ depend only on $N$ and $V$ and thus $I(N, V, \ell)$ depends only on $N, V$ and $\ell$. Finally, the condition $\delta<r_{\ell}$, which ensures that $I(N, V, \ell) \neq \emptyset$, is equivalent to

$$
0<\ell<\frac{\mathrm{d}_{\log \mathcal{O}}}{\delta}=\ell_{C}(N, V) .
$$

So, we have just proved that,

$$
\forall \ell \in\left(0, \ell_{C}\right), \forall E \in I(N, V, \ell), \forall \omega^{(0)} \in\{0,1\}^{N}, 0<\ell\left\|X_{\omega^{(0)}}(E, V)\right\| \leq \mathrm{d}_{\log \mathcal{O}} .
$$

Thus, for every $\ell \in\left(0, \ell_{C}\right)$ and every $E \in I(N, V, \ell)$,

$$
\forall \omega^{(0)} \in\{0,1\}^{N}, \ell X_{\omega(0)}(E, V) \in \log \mathcal{O} .
$$

From this, we deduce that,

$$
\forall \ell \in\left(0, \ell_{C}\right), \forall E \in I(N, V, \ell), \forall \omega^{(0)} \in\{0,1\}^{N}, T_{\omega^{(0)}}(E) \in \mathcal{O} .
$$

We actually get more from (45). As exp is a diffeomorphism from $\log \mathcal{O}$ into $\mathcal{O}$, we also have :

(47) $\forall \ell \in\left(0, \ell_{C}\right), \forall E \in I(N, V, \ell), \forall \omega^{(0)} \in\{0,1\}^{N}, \log T_{\omega^{(0)}}(E)=\ell X_{\omega^{(0)}}(E, V)$.

But, from the beginning, we choosed $V \in \mathrm{S}_{\mathrm{N}}(\mathbb{R}) \backslash \mathcal{V}$ and, by Lemma 3 , there exists $\mathcal{S}_{\mathrm{V}} \subset \mathbb{R}$ finite such that

$$
\forall E \in \mathbb{R} \backslash \mathcal{S}_{\mathrm{V}}, \operatorname{Lie}\left\{X_{\omega^{(0)}}(E, V) \mid \omega^{(0)} \in\{0,1\}^{N}\right\}=\mathfrak{s p}_{\mathrm{N}}(\mathbb{R}) .
$$

Now, by (47) and (48), as $\ell \in\left(0, \ell_{C}\right)$ is different from 0 ,

$$
\forall \ell \in\left(0, \ell_{C}\right), \forall E \in I(N, V, \ell) \backslash \mathcal{S}_{\mathrm{V}}, \operatorname{Lie}\left\{\log T_{\omega(0)}(E) \mid \omega^{(0)} \in\{0,1\}^{N}\right\}=\mathfrak{s p}_{\mathrm{N}}(\mathbb{R}) .
$$


By applying Theorem 2, we obtain that

$$
\forall \ell \in\left(0, \ell_{C}\right), \forall E \in I(N, V, \ell) \backslash \mathcal{S}_{\mathrm{V}}, G_{\{0,1\}}(E) \text { is dense in } \mathrm{Sp}_{\mathrm{N}}(\mathbb{R}) .
$$

Now, as the Fürstenberg group $G(E)$ is the closure of $G_{\{0,1\}}(E)$, we get :

$$
\forall \ell \in\left(0, \ell_{C}\right), \forall E \in I(N, V, \ell) \backslash \mathcal{S}_{\mathrm{V}}, G(E)=\mathrm{Sp}_{\mathrm{N}}(\mathbb{R}) .
$$

We have proved Proposition 1 because $\mathcal{V}$ is of Lebesgue measure 0 (see (36) and $\mathcal{S}_{\mathrm{V}}$ is finite.

We deduce Corollary 1 by using the fact that, for $\ell$ and $E$ such that $G(E)=\operatorname{Sp}_{\mathrm{N}}(\mathbb{R})$, $G(E)$ is $p$-contracting and $L_{p}$-strongly irreducible, for every $p \in\{1, \ldots, N\}$ (see [1, Definitions A.IV.3.3 and A.IV.1.1] for the definitions of these notions). Thus, by [1. Proposition IV.3.4], we get the separability and the positivity of the Lyapunov exponents $\gamma_{1}(E), \ldots, \gamma_{N}(E)$ (see (6) $)$. Because $\mathcal{S}_{\mathrm{V}}$ is finite, it is of Lebesgue measure zero in $\mathbb{R}$ and we can apply Kotani's theory (see [7]) to prove the absence of absolutely continuous spectrum in $I(N, V, \ell)$, for $\ell \in\left(0, \ell_{C}\right)$ and $V \in \mathrm{S}_{\mathrm{N}}(\mathbb{R}) \backslash \mathcal{V}$, which finish to prove Corollary 1 .

Remark 1. We also note that, by applying [2, Theorem 2], we get that the functions $E \mapsto \gamma_{p}(E)$ for $p \in\{1, \ldots, N\}$ are Hölder continuous on every compact interval $I \subset I(N, V, \ell) \backslash \mathcal{S}_{\mathrm{V}}$, for $\ell \in\left(0, \ell_{C}\right)$ and $V \in \mathrm{S}_{\mathrm{N}}(\mathbb{R}) \backslash \mathcal{V}$.

\section{Proof of Theorem 1}

Using Proposition 1 Theorem 1 will be a consequence of the following result.

Theorem 3 (Theorem 1, 3]). Let $I \subset \mathbb{R}$ be a compact interval such that $\Sigma \cap I \neq \emptyset$ and let $\tilde{I}$ be an open interval, $I \subset \tilde{I}$, such that, for every $E \in \tilde{I}, G(E)$ is $p$ contracting and $L_{p}$-strongly irreducible, for every $p \in\{1, \ldots, N\}$. Then, $H_{\ell}(\omega)$ exhibits (EL) and (SDL) in I.

To prove this result we had to :

1. Obtain an integral representation of the Lyapunov exponents of $H_{\ell}(\omega)$ which, in particular, implies their positivity.

2. Deduce from this integral representation some Hölder regularity of the Lyapunov exponents (see Remark 11).

3. Show that the integrated density of states of $H_{\ell}(\omega)$ has the same Hölder regularity (see Proposition 2).

4. Prove a Wegner estimate using the Hölder regularity of the integrated density of states.

5. Obtain (EL) and (SDL) by using multiscale analysis.

Proof of Theorem 1, Let $V \in \mathrm{S}_{\mathrm{N}}(\mathbb{R}) \backslash \mathcal{S}_{\mathrm{V}}$ and assume that $\ell \in\left(0, \ell_{C}\right)$. Let $\tilde{I} \subset$ $I(N, V, \ell) \backslash \mathcal{S}_{\mathrm{V}}$ be an open interval such that there exists $I \subset \tilde{I}$, a compact interval with $\Sigma \cap I \neq \emptyset$. If we take $\ell$ small enough, as the intervals $I(N, V, \ell)$ tends to $\mathbb{R}$, we

can always find such intervals $\tilde{I}$ and $I$. Now, as $\tilde{I} \subset I(N, V, \ell) \backslash \mathcal{S}_{\mathrm{V}}$, by Proposition 1. for every $E \in \tilde{I}, G(E)=\operatorname{Sp}_{\mathrm{N}}(\mathbb{R})$. Thus, we can apply Theorem 3 to obtain that $H_{\ell}(\omega)$ exhibits (EL) and (SDL) in $I$, which proves Theorem 1 


\section{Results ON THE INTEGRATED DENSITY OF STATES}

The integrated density of states is the distribution function of the energy levels of $H_{\ell}(\omega)$, per unit volume. To define it properly, we first need to restrict the operator $H_{\ell}(\omega)$ to finite length intervals. Let $L \geq 1$ be an integer and let $H_{\ell}^{(L)}(\omega)$ be the restriction of $H_{\ell}(\omega)$ to $L^{2}([-\ell L, \ell L]) \otimes \mathbb{R}^{N}$, with Dirichlet (or Neumann) boundary conditions at $\pm \ell L$.

Definition 4. The integrated density of states associated to $H_{\ell}(\omega)$ is the function from $\mathbb{R}$ to $\mathbb{R}_{+}, E \mapsto N(E)$, where $N(E)$, for $E \in \mathbb{R}$, is defined as :

$$
N(E)=\lim _{L \rightarrow+\infty} \frac{1}{2 \ell L} \#\left\{\lambda \leq E \mid \lambda \in \sigma\left(H_{\ell}^{(L)}(\omega)\right)\right\}
$$

for P-almost every $\omega \in \Omega$.

For the integrated density of states associated to $H_{\ell}(\omega)$, we have the following results.

Proposition 2. (1) For any $V \in \mathrm{S}_{\mathrm{N}}(\mathbb{R}), \ell>0$ and $E \in \mathbb{R}$, the limit (52) exists and is $\mathrm{P}$-almost surely independent of $\omega \in \Omega$.

(2) Let $V \in \mathrm{S}_{\mathrm{N}}(\mathbb{R}) \backslash \mathcal{V}$ and $\ell \in\left(0, \ell_{C}\right)$. Let $I \subset I(N, V, \ell) \backslash \mathcal{S}_{\mathrm{V}}$ be an open interval. Then the integrated density of states of $H_{\ell}(\omega), E \mapsto N(E)$, is Hölder continuous on $I$.

Proof. For point (1), we directly apply Corollary 1 in 2 . For point (2), we use the fact that, for $E \in I$, with $I \subset I(N, V, \ell) \backslash \mathcal{S}_{\mathrm{V}}$, we have $G(E)=\operatorname{Sp}_{\mathrm{N}}(\mathbb{R})$ and thus, $G(E)$ is $p$-contracting and $L_{p}$-strongly irreducible, for every $p \in\{1, \ldots, N\}$. As $I(N, V, \ell)$ is compact, we can directly apply to $I$ the Theorem 4 of 2 which proves that $H_{\ell}(\omega)$ is Hölder continuous on $I$.

\section{REFERENCES}

[1] P. Bougerol and J. Lacroix, Products of Random Matrices with Applications to Schrödinger Operators, Progr. Probab. Statist. 8, Birkhäuser, Boston, (1985)

[2] H. Boumaza, Hölder continuity of the integrated density of states for matrix-valued Anderson models, Rev. Math. Phys. 20(7), 873-900 (2008), DOI:10.1142/S0129055X08003456

[3] H. Boumaza, Localization for a matrix-valued Anderson model, Math. Phys. Anal. Geom. 12(3), 255-286 (2009), DOI:10.1007/s11040-009-9061-3

[4] J. Bourgain and C.E. Kenig, On localization in the continuous Anderson-Bernoulli model in higher dimension, Invent. Math. 161(2) 389-426 (2005)

[5] E. Breuillard and T. Gelander, On dense free subgroups of Lie groups, J. Algebra 261(2), 448-467 (2003)

[6] D. Damanik and R. Sims and G. Stolz, Localization for one-dimensional, continuum, Bernoulli-Anderson models, Duke Mathematical Journal 114, 59-99 (2002)

[7] S. Kotani and B. Simon, Stochastic Schrödinger operators and Jacobi Matrices on the Strip, Comm. Math. Phys. 119(3), 403-429 (1988)

E-mail address: boumaza@math.univ-paris13.fr

UMR CNRS 7539 - Département de Mathématiques, Institut Galilée, Université PARIS 13, 99 avenue Jean-Baptiste Clément, 93430 Villetaneuse, FRAnCE 